\title{
BMJ Open Protocol for a randomised controlled trial of the effect of dapagliflozin, metformin and exercise on glycaemic variability, body composition and cardiovascular risk in prediabetes (the PRE-D Trial)
}

Kristine Færch, ${ }^{1}$ Hanan Amadid,${ }^{1}$ Lea Bruhn Nielsen, ${ }^{1}$ Mathias Ried-Larsen, ${ }^{2,3}$ Kristian Karstoft, ${ }^{2}$ Frederik Persson, ${ }^{1}$ Marit Eika Jørgensen ${ }^{1,4}$

To cite: Færch K, Amadid H, Nielsen LB, et al. Protocol for a randomised controlled trial of the effect of dapagliflozin, metformin and exercise on glycaemic variability, body composition and cardiovascular risk in prediabetes (the PRE-D Trial). BMJ Open 2017;0:e013802. doi:10.1136/ bmjopen-2016-013802

- Prepublication history and additional material are available. To view these files please visit the journal online (http://dx.doi. org/10.1136/bmjopen-2016013802).

Received 8 August 2016 Revised 2 January 2017 Accepted 4 January 2017

\section{CrossMark}

${ }^{1}$ Clinical Epidemiology, Steno Diabetes Center, Gentofte, Denmark

${ }^{2}$ Centre of Inflammation and Metabolism and Centre for Physical Activity Research, Rigshospitalet, University of Copenhagen, Copenhagen, Denmark

${ }^{3}$ Danish Diabetes Academy, Odense, Denmark

${ }^{4}$ National Institute of Public Health, Southern Denmark University, Copenhagen, Denmark

Correspondence to Dr Kristine Færch; kristine.faerch@regionh.dk

\section{ABSTRACT}

Introduction The primary aim of this study is to compare the efficacy of three short-term glucose-lowering interventions (exercise, metformin and dapagliflozin) on glycaemic variability in overweight or obese men and women with elevated diabetes risk (ie, prediabetes, defined as haemoglobin $\left.\mathrm{A}_{1 \mathrm{c}}\left(\mathrm{HbA}_{1 \mathrm{c}}\right) 39-47 \mathrm{mmol} / \mathrm{mol} / 5.7 \%-6.4 \%\right)$. The secondary aims are to investigate the effects of the interventions on body composition and cardiometabolic risk factors.

Methods and analysis The Pre-D Trial is an investigatorinitiated, randomised, controlled, parallel, open-label, superiority trial. The study aims to assign 120 participants in a 1:1:1:1 ratio to receive one of four interventions for 13 weeks: (1) dapagliflozin (10 mg once daily); (2) metformin ( $850 \mathrm{mg}$ twice daily); (3) exercise (interval training,

5 days a week, 30 min per session); or (4) control (lifestyle advice). After the 13 weeks of intervention, a follow-up period of 13 weeks will follow to study the long-term effects of the interventions. The primary endpoint is reduction from baseline to end-of treatment (13 weeks) in mean amplitude of glycaemic excursions measured by continuous glucose monitoring. The secondary endpoints include concomitant changes in various measures of glucose metabolism, body weight, cardiorespiratory fitness, blood pressure, plasma lipids, objectively measured physical activity and dietary intake.

Ethics and dissemination The study protocol has been approved by the Ethics Committee of the Capital Region and the Danish Medicines Agency. Approval of data and biobank storage has been obtained from the Danish Data Protection Board. The study will be carried out according to the Declaration of Helsinki and to the regulations for good clinical practice. The results from this trial will allow a number of research questions concerning the effect of exercise versus dapagliflozin or metformin in $\mathrm{HbA}_{1 c}$-defined prediabetes to be addressed.

Trial registration NCT02695810

\section{INTRODUCTION}

Individuals with prediabetes are at high risk of developing type 2 diabetes and cardiovascular
Strengths and limitations of this study

- First randomised controlled trial testing the effect of sodium-glucose cotransporter 2 (SGLT2) inhibition in individuals with prediabetes defined by haemoglobin $A_{1}$.

- Head-to-head comparison of metformin, SGLT2 inhibitors and exercise on glycaemic variability in prediabetes.

- Detailed physiological examinations allowing investigation of energy expenditure and substrate oxidation patterns.

- The study may have limited statistical power to show interindividual differences in treatment responsiveness within each treatment arm (subgroup analyses).

disease. ${ }^{12}$ The Diabetes Prevention Program showed that lifestyle modification is more effective than metformin in lowering diabetes incidence when diabetes is diagnosed by an oral glucose tolerance test (OGTT). ${ }^{3}$ However, the superiority of lifestyle intervention over metformin is less clear when type 2 diabetes is diagnosed by haemoglobin $\mathrm{A}_{1 \mathrm{c}}$ $\left(\mathrm{HbA}_{1 \mathrm{c}}\right){ }^{4}$ This indicates that findings from individuals diagnosed by an OGTT cannot be directly transferred to individuals diagnosed by $\mathrm{HbA}_{1 \mathrm{c}}$.

The reason for the different effects of physical activity and metformin in individuals identified by OGTT versus $\mathrm{HbA}_{1 \mathrm{c}}$ may be related to differences in the relative contributions of insulin resistance and beta cell dysfunction associated with the different diagnostic criteria. Fasting hyperglycaemia is mainly caused by impaired first-phase insulin secretion and insulin resistance in the 
liver, whereas hyperglycaemia 2 hours after oral glucose ingestion is related to whole body insulin resistance and reduced second-phase insulin secretion. ${ }^{56}$ In general, prediabetes or type 2 diabetes diagnosed by $\mathrm{HbA}_{1 \mathrm{c}}$ is characterised by a combination of the defects observed in individuals with fasting versus 2-hour hyperglycaemia, ${ }^{7}$ but large differences exist between cohorts. ${ }^{89}$

$\mathrm{HbA}_{1 \mathrm{c}}$ reflects the mean glucose concentration during the past 8-12 weeks. It is well documented that high $\mathrm{HbA}_{1 c}$ levels are associated with an increased risk of diabetic complications. ${ }^{1}$ However, daily glucose fluctuations (ie, glycaemic variability) may be even more important than sustained hyperglycaemia in terms of the risk of developing diabetic complications. ${ }^{10}$ Studies have shown that glucose fluctuations increase the risk for endothelial dysfunction, retinopathy and coronary artery disease independent of the level of mean glycaemia. ${ }^{1-15}$ Also, increased use of multiple daily insulin injections and insulin pump therapy, which reduce glycaemic variability, has been associated with a reduced risk of retinopathy. ${ }^{16}$ Together, these findings suggest that reducing glucose fluctuations and not only mean glycaemia is a highly relevant focus for future diabetes-related trials. ${ }^{17}$

An often used measure of glycaemic variability is the mean amplitude of glycaemic excursions (MAGE), which reflects the mean of the differences between consecutive peaks and nadirs in blood glucose concentrations, and thereby is independent of the mean glucose level. ${ }^{15}$ MAGE is associated with coronary artery disease, vascular endothelial function and oxidative stress independent of $\mathrm{HbA}_{1 \mathrm{c}}$ and fasting plasma glucose levels. ${ }^{11-14}$ Different interventions are expected to have different effects on mean, fasting and postprandial glucose concentrations, as well as on MAGE. Exercise is known to reduce the postprandial glucose response $\mathrm{e}^{18-21}$ and thereby will reduce MAGE. ${ }^{22}$ It is recommended that patients with type 2 diabetes as well as persons with prediabetes should perform at least $150 \mathrm{~min}$ of moderate-to-vigorous intensity aerobic exercise per week. ${ }^{23} \mathrm{~A}$ recent study in adults with a high risk of type 2 diabetes suggests that to obtain an improvement in glycaemic control, exercise should be performed in sessions with high intensities as compared with sessions with a longer duration with lower intensities (same total energy expenditure). ${ }^{24}$ In line with this, several studies suggest that interval training has a favourable effect on glycaemic control and glycaemic variability in persons with or at high risk of type 2 diabetes and can be performed with a high compliance. ${ }^{25-29}$

In contrast to exercise, the actions of metformin are predominantly on hepatic insulin sensitisation and inhibition of gluconeogenesis. Metformin will therefore preferentially lower fasting glucose concentrations $^{330-34}$ and is not likely to have the same beneficial effects on MAGE as exercise. Sodium-glucose cotransporter 2 (SGLT2) inhibitors are a relatively new class of medication for patients with type 2 diabetes. SGLT2 inhibitors block glucose reabsorption in the kidney if blood glucose levels exceed $9-10 \mathrm{mmol} / \mathrm{L}^{35}$.
Their efficacy and safety have been studied in multiple randomised controlled trials of individuals with type 2 diabetes. ${ }^{36-38}$ Treatment with SGLT2 inhibitors has shown to lower the renal threshold for glucose excretion in a dose-dependent manner both in individuals with type 2 diabetes and normal glucose regulation. ${ }^{39} 40$ In normal glucose-tolerant normal-weight to overweight men, SGLT2 inhibitors reduce postprandial glucose and insulin levels by increasing urinary glucose excretion. ${ }^{41}$ However, fasting glucose levels do not seem to be affected by SGLT2 inhibition in non-diabetic individuals. ${ }^{42}$ Because SGLT2 inhibitors are mostly effective at blood glucose levels above $9-10 \mathrm{mmol} / \mathrm{L}^{35}$, whereas physical activity and exercise can reduce postprandial glucose levels even in the normal and prediabetic glycaemic range, ${ }^{19}{ }^{43}$ it is expected that the exercise intervention is superior to SGLT2 inhibitors in reducing MAGE.

To date, metformin is the only widely accepted medication for use in prediabetes. ${ }^{44}$ This trial will provide evidence as to whether it will be relevant to further examine the long-term effect of SGLT2 inhibitors on prevention of type 2 diabetes and cardiovascular disease in prediabetic individuals.

\section{OBJECTIVES}

The primary objective of this study is to compare the short-term (13 weeks) efficacy of three glucose-lowering interventions (exercise, metformin and SGLT2 inhibition) on glycaemic variability in overweight or obese individuals with prediabetes defined as $\mathrm{HbA}_{1 \mathrm{c}} 39-47 \mathrm{mmol} / \mathrm{mol}(5.7 \%-6.4 \%)$. The secondary aims are to investigate the effects of the interventions on body composition and cardiometabolic risk factors. Third, we aim to identify subgroups of individuals who do not respond or respond better than others to lifestyle and pharmacological interventions, and to determine how daily exercise bouts and time spent sedentary and in moderate-to-vigorous physical activity intensities are related to measures of glycaemic variability, insulin sensitivity and beta cell function.

\section{HYPOTHESES}

In relation to the overall objective, the following are the hypotheses:

1. Exercise is superior to the pharmacological interventions in reducing glycaemic variability.

2. Metformin will predominantly lower fasting glucose levels and only have minor effects on postprandial glucose levels. Dapagliflozin will reduce glucose levels in the postprandial state, but not in the fasting state. Accordingly, dapagliflozin is superior to metformin in reducing glycaemic variability. 


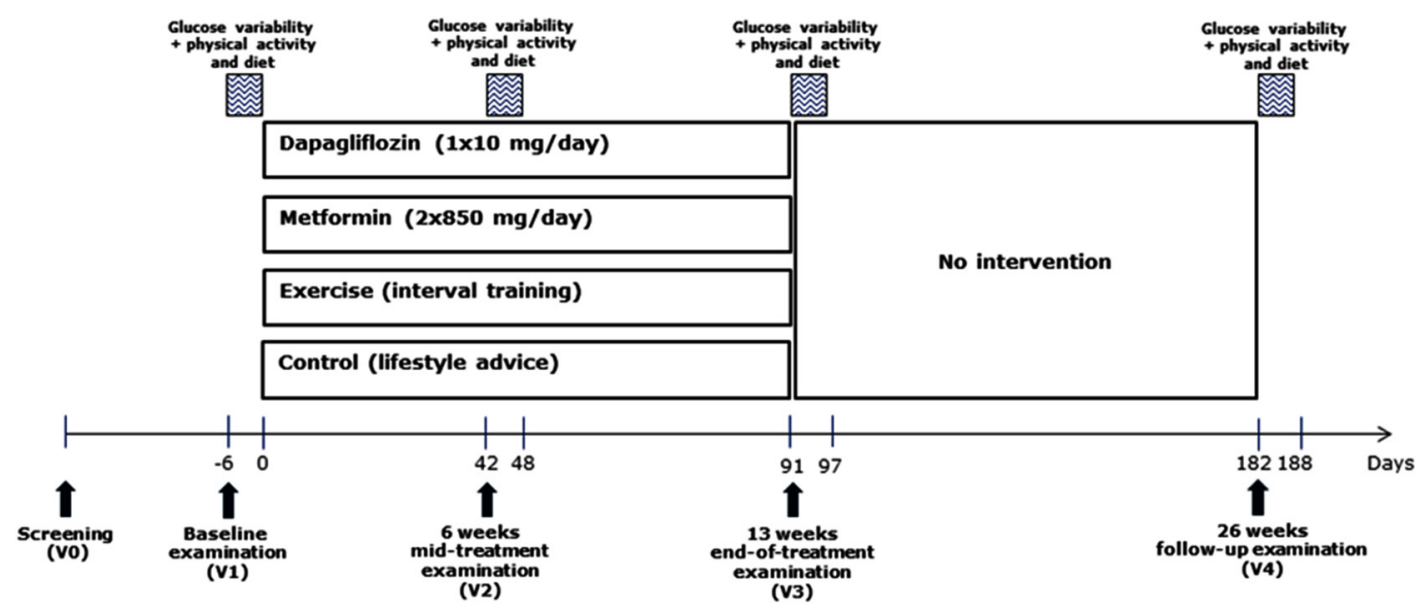

Figure 1 Design of the randomised controlled trial.

\section{METHODS AND ANALYSIS Study design}

The study is an investigator-initiated, randomised, controlled, parallel, open-label trial (figure 1). A total of 120 participants will be randomly assigned in a 1:1:1:1 ratio to receive one of four interventions for 13 weeks: (1) dapagliflozin (10 mg once daily); (2) metformin (850 mg twice daily); (3) exercise (interval training, 5 days a week, 30 min per session); or (4) control (lifestyle advice). After the 13 weeks of intervention, a follow-up period of 13 weeks will follow to study the longer term effects of the interventions.

\section{Participants}

Eligible participants are overweight or obese adults who meet the eligibility criteria for prediabetes defined by $\mathrm{HbA}_{1 \mathrm{c}}^{45}$ (box 1). After informed consent, participants who meet the inclusion criteria without conditions leading to exclusion at the screening examination will be enrolled for randomisation, followed by 13 weeks of treatment and 13 weeks of follow-up at the Steno Diabetes Center, Gentofte, Denmark. Inclusion and exclusion criteria are listed in box 1.

\section{Eligibility criteria}

Although recruitment is mainly directed at middle-aged overweight individuals, the age criterion was set at $\geq 30$ years to include groups at high risk for prediabetes in early adulthood, such as young women with a history of gestational diabetes. An upper age limit of 70 years is set because the ability to reduce the risk or postpone the development of diabetes and cardiovascular disease is expected to be limited in individuals above 70 years of age. The body mass index (BMI) criterion of $\geq 25 \mathrm{~kg} / \mathrm{m}^{2}$ is used in order to identify individuals with a high risk of having prediabetes, thereby limiting the number of screen failures.

Most exclusion criteria are chosen to reduce the risk of adverse effects related to the interventions. Individuals with clinically significant cardiovascular or pulmonary diseases are excluded because the examinations require performing cardiorespiratory fitness tests and the exercise intervention requires performing interval training in alternating high and low intensities. Also individuals with injuries or disabilities that make them unable to perform the interval training are excluded. At the screening, all participants will be asked about their motivation to participate in the trial if they are randomised to exercise,

\section{Box 1 Inclusion and exclusion criteria}

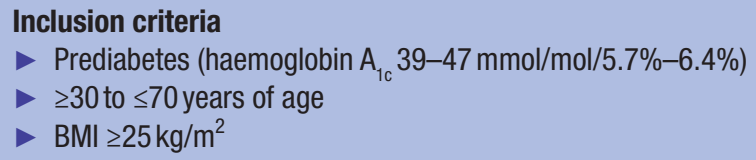

\section{Exclusion criteria}

- Uncontrolled medical issues including but not limited to cardiovascular pulmonary, rheumatological, haematological, oncological, infectious, gastrointestinal or psychiatric disease; diabetes or other endocrine disease; immunosuppression

- Current treatment with hormones that affect glucose metabolism

- Current treatment with loop diuretics or thiazide diuretics

- Current treatment with beta blockers or peroral steroids

- Bariatric surgery within the past 2 years

- Impaired renal function defined as an estimated GFR<60 mL/ $\min / 1.73 \mathrm{~m}^{2}$

- Neurogenic bladder disorders

- Alcohol/drug abuse or in treatment with disulfiram (Antabus) at time of inclusion

Pregnant or lactating women

- Fertile women not using birth control agents, including oral contraceptives, gestagen, injection, subdermal implantation hormonal vaginal ring, transdermal application or intrauterine devices

- Allergic to one or more of the medications used in the study

- Unable to exercise according to protocol (judged by investigator based on the participant's motivation, injuries/disabilities, and evaluation of ECG)

- Concomitant participation in other intervention study

- Unable to understand the informed consent and the study procedures

BMI, body mass index; GFR, glomerular filtration rate. 
medication or the control group in order to lower the risk of dropouts after randomisation.

Individuals with any degree of renal impairment are excluded because of their increased risk of adverse events with both metformin ${ }^{46}$ and dapagliflozin ${ }^{47}$ treatments. In addition, the efficacy of dapagliflozin on glucose reabsorption is limited in individuals with impaired renal function. ${ }^{47}$ Pregnant or nursing women as well as women who anticipate pregnancy during the course of the programme are also excluded from the study. The reason is that in Denmark neither metformin nor dapagliflozin is recommended during pregnancy or nursing. In addition, pregnancy modifies insulin resistance, which can affect the outcome measures.

Thiazide diuretics, loop diuretics and beta blockers are commonly used to treat hypertension, which often coexists with prediabetes. Dapagliflozin may add to the diuretic effect of thiazide and loop diuretics and may increase the risk of dehydration and hypotension. Because these agents may cause adverse events in relation to the interventions, individuals using thiazides, loop diuretics or beta blockers on a daily basis are ineligible. However, such individuals may be included in the study if they meet $\mathrm{HbA}_{1 \mathrm{c}}$ and other eligibility criteria after their treatment is changed to other antihypertensive drugs without known adverse effects related to the interventions.

\section{Recruitment of participants}

Recruitment strategies appropriate for the identified target population include advertisements in online media and newspapers, recruitment of relatives to patients with diabetes at Steno Diabetes Center, and recruitment through contact with local general practitioners. Persons who show their interest in participating will be approached by a member of the research team to screen for preparticipation eligibility and explain the main requirements of participating in the study. Individuals are considered eligible at this step if the age and BMI criterion is met, and written information is sent to the potential participant. Those who are interested in participating after reading the material are invited to a health examination and screening (V0, table 1). Participants who are eligible after the screening are included in the study (V1-V4, table 1).

\section{Endpoints}

\section{Primary endpoint}

The primary endpoint is changes in MAGE from baseline to end-of treatment (13 weeks). MAGE will be estimated from the 6-day sensor glucose profiles by a researcher blinded to the interventions. MAGE will be calculated by taking the arithmetic mean of the blood glucose increases or decreases (from blood glucose nadirs to peaks or vice versa) when both ascending and descending segments exceed the value of $1 \mathrm{SD}$ of the blood glucose during a 24-hour measurement period. ${ }^{15}$

\begin{tabular}{llllll} 
Table 1 & Schematic overview of study visits \\
\hline Visit & V0 & V1 & V2 & V3 & V4 \\
\hline $\begin{array}{l}\text { Time, days from } \\
\text { intervention start }\end{array}$ & $-90^{*}$ & -6 & 42 & 91 & 182
\end{tabular}

Participant-related

information

\begin{tabular}{|c|c|c|c|c|c|}
\hline Informed consent & $x$ & & & & \\
\hline History & $\mathrm{X}$ & & & & \\
\hline $\begin{array}{l}\text { Inclusion/Exclusion } \\
\text { criteria }\end{array}$ & $x$ & & & & \\
\hline Clinical examination & & $x$ & $x$ & $x$ & $x$ \\
\hline \multicolumn{6}{|l|}{$\begin{array}{l}\text { Efficacy and safety } \\
\text { outcomes }\end{array}$} \\
\hline $\mathrm{HbA}_{1 \mathrm{C}}$ & $x$ & $x$ & $x$ & $x$ & $x$ \\
\hline Body weight & $\mathrm{X}$ & $\mathrm{X}$ & $x$ & $x$ & $x$ \\
\hline Height & $\mathrm{X}$ & & & & \\
\hline Waist circumference & & $x$ & $x$ & $x$ & $x$ \\
\hline Blood pressure & & $X$ & $X$ & $x$ & $\mathrm{X}$ \\
\hline Pregnancy test & $x$ & $(X)$ & $(X)$ & $(X)$ & $(X)$ \\
\hline Fasting blood samples & & $\mathrm{X}$ & $X$ & $x$ & $x$ \\
\hline Urine samples & & $x$ & $x$ & $x$ & $x$ \\
\hline Indirect calorimetry & & $X$ & & $X$ & $\mathrm{X}$ \\
\hline $\begin{array}{l}\text { Oral glucose tolerance } \\
\text { test }\end{array}$ & & $x$ & & $x$ & $x$ \\
\hline $\begin{array}{l}\text { Body fat distribution } \\
\text { (DEXA) }\end{array}$ & & $x$ & & $x$ & $x$ \\
\hline Fitness test & & $x$ & & $x$ & $x$ \\
\hline $\begin{array}{l}\text { Free-living physical } \\
\text { activity measurement }\end{array}$ & & $x$ & $x$ & $x$ & $x$ \\
\hline $\begin{array}{l}\text { Continuous glucose } \\
\text { monitoring }\end{array}$ & & $x$ & $x$ & $x$ & $x$ \\
\hline Adverse events & & $x$ & $x$ & $x$ & \\
\hline \multicolumn{6}{|l|}{ Questionnaires } \\
\hline Health and well-being & & $x$ & $x$ & $x$ & $x$ \\
\hline Physical activity & & $x$ & $x$ & $x$ & $x$ \\
\hline Sleep patterns & & $x$ & $x$ & $x$ & $x$ \\
\hline Food diary & & $x$ & $x$ & $x$ & $x$ \\
\hline Treatment satisfaction & & & $x$ & $\mathrm{X}$ & \\
\hline $\begin{array}{l}\text { Study medication, drug } \\
\text { accountability }\end{array}$ & & & $\underline{x}$ & $\underline{x}$ & \\
\hline
\end{tabular}

*The maximum allowed time from the screening (V0) to the baseline examination (V1) is 12 weeks (=84 days). If this is not possible, a new screening will be performed before including the participant in the study.

HbA1c, haemoglobin $A_{1 c}$; DEXA, dual-energy X-ray absorptiometry.

\section{Secondary endpoints}

The secondary endpoints include changes from baseline to mid-of-treatment (6 weeks), end-of-treatment (13 weeks) and follow-up (26 weeks) in the following parameters: $\mathrm{HbA}_{1 \mathrm{c}}$, daily time spent above different 
glucose concentrations $(\mathrm{eg},>6.1 \mathrm{mmol} / \mathrm{L},>7.0 \mathrm{mmol} / \mathrm{L}$, $>7.8 \mathrm{mmol} / \mathrm{L}$ and $>11.1 \mathrm{mmol} / \mathrm{L})$, glucose concentrations during OGTT (0, 30, 60 and $120 \mathrm{~min})$, Insulinogenic Index, Insulin Sensitivity Index, body weight, body fat distribution, cardiorespiratory fitness, basal energy expenditure, substrate oxidation patterns, time spent sedentary and in moderate-to-vigorous physical activity intensity, blood pressure, heart rate, plasma lipids, biomarkers of metabolic functions, number of adverse events and side effects, changes in dietary habits, sleep patterns, self-rated health and quality of life, and adherence to the different interventions.

\section{Study visits and examinations}

No study-related procedures will take place before informed consent has been obtained after careful written and oral information to the participant about the study. Individuals who agree to participate in the study will be invited to a screening visit (V0). All examinations will be performed at Steno Diabetes Center. Study visits and examinations are summarised in table 1 . The examinations are described below.

\section{Clinical examination}

A clinical examination including measurement of body weight, waist and hip circumference, blood pressure, and pulse is performed at all visits. Height is only measured at the screening visit. Height and body weight will be measured with the participant wearing light indoor clothes and no shoes. Waist circumference is measured halfway between the lowest point of the costal margin and highest point of the iliac crest, and hip circumference is measured at the level of the greater femoral trochanter; both are measured to the nearest $0.5 \mathrm{~cm}$. Measurements of waist and hip circumference are performed three times each. Blood pressure and pulse are measured with the participant in sitting position after a minimum $10 \mathrm{~min}$ of rest using a digital blood pressure monitor, Model UA-852 (A\&D Instruments, Oxfordshire, UK). Blood pressure and pulse measurements are repeated three times separated by 2 min breaks. The mean value of the last two measurements is used.

\section{Continuous glucose monitoring}

A 6-day continuous glucose monitoring for assessment of glycaemic variability will be performed at V1, V2, V3 and V4. The iPro2 Continuous Glucose Monitoring System (CGMS) will be used (Medtronic Danmark A/S, Copenhagen, Denmark). The CGMS sensor will be inserted in the subcutaneous adipose tissue on the lower part of the abdomen (under the umbilicus). In order to calibrate the CGMS, participants will monitor home glucose levels before breakfast, before lunch, before main evening meal and before bedtime using a glucometer (Contour XT, Ascensia Diabetes Care Denmark ApS, Copenhagen, Denmark).
Free-living physical activity and dietary intake

Concomitant with the 6-day measurement of glycaemic variability, measurement of free-living physical activity will be performed. Free-living physical activity energy expenditure will be measured with accelerometers (Axivity AX3, Newcastle upon Tyne, UK). The participants will wear two accelerometers (one on the thigh and one on the back) for 6 days. Participants will be instructed to send the accelerometers and the continuous glucose monitor back to the investigator by postage after the 6 days of measurements. During the first 4 days of measurement of physical activity and continuous glucose monitoring, the participants are asked to register their entire intake of food and caloric beverages (grams per portion and time of ingestion).

\section{Electrocardiogram}

An ECG is performed at the baseline visit and at the visits after 13 and 26 weeks in order to screen for heart conditions, which may exclude participants from performing a maximal fitness test.

\section{Indirect calorimetry}

The respiratory exchange ratio will be measured by indirect calorimetry after standardised conditions, including $>8$ hours of fasting and no exercise for 48 hours. The measurement takes place in a quiet room, where the participant is placed in supine position. The respiratory exchange ratio is calculated from the relationship between oxygen consumption and carbon dioxide production and will be used as a measure of the relative contributions of carbohydrate and lipid oxidation in the basal state. The measurement is performed with a ventilated hood using a JAEGER Oxycon Pro analyser (Erich JAEGER GmbH, Hoechberg, Germany). Before each measurement, the equipment is flow-calibrated and gas-calibrated to take into account subtle changes in humidity, temperature and content of $\mathrm{O}_{2}$ and $\mathrm{CO}_{2}$ in the examination room.

\section{Oral glucose tolerance test}

The participant is instructed to fast (water is allowed) for 8-10 hours prior to the tests. A small venous catheter will be inserted in one of the participant's arms for blood sampling. The participant will drink a $200 \mathrm{~mL}$ glucose solution (75 g glucose) within $1 \mathrm{~min}$. Blood samples will be drawn at 0, 30, 60 and $120 \mathrm{~min}$ for assessment of plasma glucose, insulin, C peptide, glucagon, glucagon-like peptide-1 and glucose-dependent insulinotropic polypeptide.

\section{Body fat content}

A dual-energy X-ray absorptiometry (DEXA) scan will be performed to measure body fat content. Participants will lie still on a table while a machine arm passes over their entire body, which emits a high-energy and a low-energy X-ray beam. By measuring the absorption of each beam into parts of the body, readings for bone mineral density, lean body mass and fat mass will be obtained. 


\section{Cardiorespiratory fitness}

After the OGTT, a fitness test for determination of peak oxygen consumption $\left(\mathrm{VO}_{2 \text { peak }}\right)$ and peak heart rate $\left(\mathrm{HR}_{\text {peak }}\right)$ will be performed. The test will be performed as an incremental test on a cycle ergometer (Monark LC4) with indirect calorimetry (JAEGER Oxycon Pro analyser, Erich JAEGER GmbH). Study participants will be cycling for $6 \mathrm{~min}$ as a warm-up ( $3 \mathrm{~min}$ at $30 \mathrm{~W}$ and $3 \mathrm{~min}$ at $60 \mathrm{~W}$ for women; $3 \mathrm{~min}$ at $40 \mathrm{~W}$ and $3 \mathrm{~min}$ at $80 \mathrm{~W}$ for men). After the warm-up, the workload is increased every minute by $20 \mathrm{~W}$ for women and $25 \mathrm{~W}$ for men until exhaustion.

\section{Questionnaires}

Self-reported physical activity is measured using the recent physical activity questionnaire. This questionnaire assesses physical activity across four domains (domestic, recreational, work, commuting) over the previous month. Questionnaires on health and well-being, sleep patterns and satisfaction with the interventions will also be filled out at each visit at the study site. During the 6-day CGM measurements, the study participants will be asked to fill in a diary describing the type and duration of physical activity. In addition, the participants are asked to weigh and register intake of meals and caloric beverages consumed during the first 4 days of the CGMS measurement period.

\section{Sociodemographic information}

Baseline sociodemographic information, which could act as covariates or confounders for the tested interventions, will also be collected. These include age, ethnicity, civil status, education, occupation, health history, smoking status and alcohol consumption.

\section{Interventions}

\section{Dapagliflozin}

Dapagliflozin (10 mg) will be administered once daily as monotherapy. The product will be delivered by Gentofte Apotek and labelled by the investigator at Steno Diabetes Center. Common side effects $(1 \%-10 \%)$ include low blood pressure, increased number of red blood cells, change in plasma lipid profile, back pain, dizziness, inflammation in and around the vagina, inflammation of the foreskin, urinary tract infection, painful urination and increased urine volume.

\section{Metformin}

Metformin $(850 \mathrm{mg}$ ) will be administered twice daily as monotherapy (1 tablet together with breakfast, 1 tablet together with dinner). Titration will be performed according to the guidelines for patients with type 2 diabetes (one tablet daily the first week, then two tablets daily for the rest of the treatment period). Metformin will be delivered by Gentofte Apotek and labelled by the investigator at Steno Diabetes Center. Common side effects (1\%-10\%) include decreased appetite, nausea, vomiting, diarrhoea, stomach pain and dysgeusia.

\section{Exercise}

The exercise intervention will consist of interval training 5 days a week, $30 \mathrm{~min}$ per session, at alternating $3 \mathrm{~min}$ intervals aiming at reaching intensities of $\geq 75 \%$ (high) and $\leq 60 \%$ (low) of $\mathrm{HR}_{\text {peak }}$ by the end of each interval. If the participants experience challenges in reaching these intensities, they are advised to aim for at least $15 \%$ difference between the high and low intervals (eg, 80\% and $65 \%$ of $\mathrm{HR}_{\text {peak }}$ ). The participants can freely choose between walking, cycling, running or other aerobic activities, and they can do the activities outdoor or in a fitness centre. They will be offered membership to a local fitness centre for the entire intervention period. The exercise will be monitored, evaluating the heart rate response of all exercise sessions. The participants will record the heart rates and durations of exercise bouts using Polar V800 (Polar Electro, Kempele, Finland). The participants will be asked to upload heart rate data from the Polar watches to the software Polar Flow on their computer twice a week. Computer-generated reminders are sent to the participants twice a week to ensure compliance on the upload. As feedback and encouragement are important factors in adherence, ${ }^{48}$ the investigator will provide encouraging feedback in the form of short written comments and 'likes' to the participants twice a week after upload of data using the online tool 'Polar Flow for Coach' (Polar Electro Danmark ApS, Holte, Denmark). Additionally, an e-mail is sent out to the participants once weekly with a short status report on the participant's compliance to exercise during the past week. In case of technical challenges related to the watch or heart rate monitor, the participant is contacted by telephone to solve the problem.

Within the first week after randomisation, the participants are invited to an introduction to the exercise intervention at Steno Diabetes Center. During this introduction, a thorough training on the use of heart rate monitor and watch will take place in order to teach the participants how to exercise at the alternating intensities and make them familiar with the technology used.

\section{Control}

At the baseline examination, the control group as well as the three other groups will receive oral and written information about a healthy lifestyle and weight loss according to the official dietary recommendations from the Danish Veterinary and Food Administration. ${ }^{49}$

\section{Randomisation}

For safety and practical reasons, randomisation will be open for both participants and investigators, but assessment of the primary outcome is blinded. Randomisation codes have been produced by the sponsor by use of the web-based Clinical Trial Management System EasyTrial (EasyTrial ApS, Glostrup, Denmark). ${ }^{50}$ Randomisation is performed in blocks in order to secure an equal distribution of participants in each intervention group if the trial unexpectedly will be terminated before inclusion of the planned number of participants. Details of the size of the 
blocks are unavailable to the investigators performing the examinations and allocating participants to the interventions. Allocation to intervention groups will take place at the end of the baseline examination (at day -6) to secure that the investigator performs all baseline measurements unbiased of the allocated intervention. After the baseline measurements and allocation, the investigator puts the allocated intervention into a box (metformin tablets, dapagliflozin tablets, a Polar watch or a carton filled with paper), and the box is locked with a coded lock. All boxes have identical looks. The participant then receives the box containing his/her allocated treatment regime, but it is not revealed for the participant what the box contains. Six days after the baseline examination (at day 0 ), the participant will receive the code to the box by telephone from the investigator. This procedure is done in order to secure that the 6-day baseline measurements of continuous glucose monitoring and physical activity are performed unbiased of the allocated intervention by the participants.

\section{Assessment of compliance}

\section{Medication}

Participants receiving medication are asked to bring their study medication to visit 2 (6 weeks) and visit 3 (13 weeks). The investigator will register the number of tablets taken and thereby assess compliance to the medication. A compliance of at least $80 \%$ is considered satisfactory.

\section{Exercise}

For participants in the exercise intervention group, compliance is monitored online via Polar V800 (Polar Electro, Finland) and the online tool 'Polar Flow for Coach'. Participants completing $\geq 80 \%$ of the training volume prescribed (ie, 120 min per week) and $\geq 80 \%$ of the exercise sessions prescribed (ie, four sessions per week) are considered to be compliant. To prevent dropouts, two procedures are implemented if a participant expresses concerns about compliance with the prescribed exercise intervention or if the investigators experience that a participant does not follow the prescribed exercise intervention (eg, upload of exercise sessions is lacking, or the duration or intensity of the exercise bouts is not in compliance with the protocol):

1. Maintenance of training volume: The participant is offered a telephone interview to identify possible challenges in relation to the exercise intervention, that is, lack of time or worries. An adjusted plan is made in collaboration between the investigator and participant with the aim of maintaining the weekly training volume as per protocol, for example, fewer sessions of exercise per week but with longer duration (unchanged intensity).

2. Reducing training volume: If training volume cannot be maintained (eg, in case of injury or other personal issues), up to two exercise sessions per week are eliminated from the programme for 2 weeks or more if necessary.

\section{ETHICS, SAFETY, DATA MANAGEMENT AND DISSEMINATION}

This intervention study will provide important information about the effect of exercising versus prescribing dapagliflozin or metformin therapy to individuals with prediabetic glucose levels defined by the $\mathrm{HbA}_{1 \mathrm{c}}$ criteria. All equipment used in the study meet the requirements for patient safety. For the determination of body composition, DEXA scanning with a weak X-ray radiation is used. The radiation dose is less than $0.01 \mathrm{mSv}$, which corresponds to less than 1 day of normal background radiation. The dapagliflozin tablets contain lactose, which may cause discomfort in lactose-intolerant individuals. Dapagliflozin and metformin are not yet registered for treatment of individuals with prediabetes in Denmark. Side effects such as low blood pressure, increased number of red blood cells, change in plasma lipid profile, back pain, dizziness, inflammation in and around the vagina, inflammation of the foreskin, urinary tract infection, painful urination, increased urine volume, decreased appetite, nausea, vomiting, diarrhoea, stomach pain and dysgeusia can be expected in some study participants. However, it is believed that the potential beneficial effects of dapagliflozin and metformin on glycaemic control will counterbalance the potential unfavourable effects. ${ }^{51}{ }^{52}$ The participants are covered by the Patient Compensation Association according to the Danish Act on the Right to Complain and Receive Compensation within the Health Service.

The study protocol is approved by the Ethics Committee of the Capital Region (H-15011398) and the Danish Medicines Agency (EudraCT number: 2015-001552-30). Approval for data storage has been obtained from the Danish Data Protection Board (2012-58-0004). The study is registered with ClinicalTrials.gov (NCT02695810) and will be carried out in accordance with the Declaration of Helsinki II and to the regulations for good clinical practice (GCP). The unit for GCP in Copenhagen University Hospital will perform audit site visits in order to secure that the study is performed in accordance with the International Council for Harmonisation (ICH)-GCP guidelines.

The investigator is responsible for ensuring that all serious adverse reactions/adverse events are immediately reported to the sponsor, who will then notify the Ethics Committee of the Capital Region and the Danish Medicines Agency according to the existing laws and ICH-GCP guidelines. In case of unexpected severe adverse reactions to medication during the study, the trial will be discontinued. In case participants suffer harm from participation in this trial, they will be referred to the Patient Compensation Association.

The web-based Clinical Trial Management System EasyTrial is used for data entry and management (EasyTrial ApS).$^{50}$ EasyTrial has been approved by the Danish Data Protection Board. Electronic case reports forms and questionnaires have been generated by the sponsor in EasyTrial. Fields have been programmed with acceptable ranges for data entry. EasyTrial is also used to send 
reminders to the participants prior to visits and to remind participants in the exercise intervention group to upload heart rate data for supervision. During the study, data are entered directly into the system by the investigators, and after study completion data will be extracted directly from the system by the sponsor/investigators.

Positive, negative and inconclusive study results will be published by the investigators in international peer-reviewed journals and presented at international conferences. Manuscripts will be written in accordance with the online supplementary CONSORT statement and the Vancouver Declaration.

\section{STATISTICAL METHODS}

\section{Sample size considerations}

MAGE has not previously been used as primary outcome in randomised controlled trials of persons with prediabetes. A study has shown that mean (SD) MAGE is significantly higher in individuals with impaired fasting glycaemia (IFG) and (IGT) $(2.26(0.7) \mathrm{mmol} / \mathrm{L})$ than in people with normal glucose tolerance $(1.60(0.7)$ $\mathrm{mmol} / \mathrm{L}$ ) despite having similar $\mathrm{HbA}_{1 \mathrm{c}}$ levels (5.7 vs $5.5 \%) .{ }^{53}$ Similarly, MAGE was found to be significantly higher (mean (SD) MAGE: $2.7(0.4) \mathrm{mmol} / \mathrm{L}$ ) in women with previous gestational diabetes (mean $\mathrm{HbA}_{1 \mathrm{c}} 5.8 \%$ ) than in weight-matched normal glucose-tolerant women without a history of gestational diabetes (mean (SD) MAGE: $1.8(0.5) \mathrm{mmol} / \mathrm{L}$; mean $\left.\mathrm{HbA}_{1 \mathrm{c}} 5.4 \%\right) .{ }^{54}$ In addition, a Chinese study found mean (SD) MAGE to be 2.1 (0.8) $\mathrm{mmol} / \mathrm{L}$ in 23 abdominally obese men versus 1.6 (0.5) $\mathrm{mmol} / \mathrm{L}$ in 23 non-abdominally obese men with normal glucose regulation $(\mathrm{p}<0.05) .^{55}$

Randomised controlled trials in patients with type 2 diabetes (mean duration of diabetes $\sim 5$ years) found that MAGE decreased from mean (SD) 4.9 (1.0) to 3.7 (0.9) $\mathrm{mmol} / \mathrm{L}$ after treatment with metformin + sitagliptin $(\mathrm{p}<0.001) .{ }^{56}$ The mean $(\mathrm{SD})$ decrease in MAGE after 15 weeks of metformin treatment in patients with newly diagnosed type 2 diabetes was 1.4 (1.6) mmol/ $\mathrm{L}^{57}$. In terms of SGLT2 inhibition, a study of 15 patients with type 1 diabetes found a mean $(95 \% \mathrm{CI})$ reduction of 3.8 ( 1.5 to 6.1$) \mathrm{mmol} / \mathrm{L}$ in MAGE after 7 days of treatment with dapagliflozin. ${ }^{58}$ In relation to exercise, a single bout of exercise has shown to decrease average blood glucose by $\sim 0.9 \mathrm{mmol} / \mathrm{L}$ in patients with type 2 diabetes - also in those with $\mathrm{HbA}_{1 \mathrm{c}}$ levels below $7 \% .^{22}$ In another study of patients with type 2 diabetes, 2 weeks of interval walking reduced MAGE by $1.8(0.5) \mathrm{mmol} / \mathrm{L}^{29}$

It is expected that both mean and SD of MAGE are lower among individuals with prediabetes than in individuals with type 2 diabetes. With a power of $80 \%$ (alpha level of 0.05 ) in a two-sided test, a clinically meaningful difference in the change in MAGE over the 13-week intervention of $\geq 0.5 \mathrm{mmol} / \mathrm{L}$ (SD: $0.6 \mathrm{mmol} / \mathrm{L}$ ) between two groups can be found with 23 participants in each group. To allow for dropouts ( $20 \%)$ and subgroup analyses, we plan to include 30 participants in each of the four study
Table 2 Sample size calculations based on a power of 0.8 and an alpha level of 0.05 in a two-sided test with different $\mathrm{SD}$ and mean differences in MAGE

\begin{tabular}{llll}
\hline Scenario & $\begin{array}{l}\text { Mean } \\
\text { difference }\end{array}$ & SD & $\begin{array}{l}\text { Participants needed } \\
\text { in each group (n) }\end{array}$ \\
\hline 1 & 0.5 & 0.7 & 31 \\
2 & 0.5 & 0.6 & 23 \\
3 & 0.5 & 0.5 & 16 \\
4 & 0.6 & 0.7 & 22 \\
\hline 5 & 0.6 & 0.6 & 16 \\
6 & 0.6 & 0.5 & 11 \\
\hline 7 & 0.7 & 0.7 & 16 \\
8 & 0.7 & 0.6 & 12 \\
9 & 0.7 & 0.5 & 8 \\
\hline
\end{tabular}

groups. Inclusion of participants is terminated when 120 participants have been included. In case fewer than 120 participants are recruited, the minimum mean differences in MAGE expected to be statistically significant are shown in table 2 (with different $\mathrm{SD}$ ).

Participants who withdraw from the study will not be replaced. Participants who are excluded or who decide to stop their participation will be referred to their general practitioner for advice on how to manage their elevated diabetes risk. Data on withdrawn participants will be collected at the end of the study and used in the safety analysis if allowed by the participant.

\section{Statistical analysis}

Intention-to-treat (ITT) analysis will be performed after the last participant has completed the last visit. In addition, per-protocol analyses will be made $(\geq 80 \%$ compliance to the interventions). In principle, the ITT strategy requires a complete follow-up of all randomised participants for study outcomes, which may not be possible to achieve, and therefore the ITT analysis may give a biased estimate of the treatment effect. On the other hand, the ITT analysis reflects treatment in clinical practice to a higher degree than per-protocol analyses, which only include people who are compliant to the interventions. In order to address these issues, analyses with imputation of missing data will be performed. Patterns of missing data will be investigated. It is expected that missing data will be 'missing at random' rather than 'missing completely at random', because it is assumed that dropouts may depend on observed outcomes or covariates but not on unobserved data. Subgroup analyses stratified by age, sex, obesity degree and prediabetic subgroup (fasting vs 2-hour hyperglycaemia) will also be performed.

Parametric tests (general linear models) will be used to test differences in outcomes from baseline to follow-up. If model assumptions cannot be met even after logarithmic transformation, non-parametric tests will be used. Plots of residuals versus predicted values will be used to judge normality. Two-sided tests will be used and $\mathrm{p}$ 
values of $<0.05$ are considered significant. Adjustment for multiple testing will be performed.

\section{DISCUSSION}

The guidelines from the American Diabetes Association ${ }^{45}$ suggest that individuals with prediabetes (ie, IGT, IFG or $\mathrm{HbA}_{1 \mathrm{c}} 39-47 \mathrm{mmol} / \mathrm{mol} / 5.7 \%-6.4 \%$ ) should increase physical activity level to at least $150 \mathrm{~min} /$ week and lose body weight. In prediabetic individuals who are $<60$ years of age, have BMI $>35 \mathrm{~kg} / \mathrm{m}^{2}$ or have a history of gestational diabetes, metformin therapy should be considered. Furthermore, the American Diabetes Association states that aggressive interventions should be pursued for those considered at very high risk (eg, those with $\mathrm{HbA}_{1 \mathrm{c}}>42$ $\mathrm{mmol} / \mathrm{mol} / 6.0 \%) .{ }^{45}$ However, the evidence behind these recommendations is predominantly based on individuals with IGT; evidence underlying a strategy for $\mathrm{HbA}_{1 c}$-defined prediabetes is lacking.

Rigorous monitoring of exercise bouts using online technology will provide experience and may constitute a new way of conducting studies of real-life physical activity interventions. Our study will describe glycaemic variability and a range of metabolic parameters in prediabetes, giving us the opportunity to determine detailed characteristics of individuals at risk, potentially identifying new parameters for interventions or prediction of future diseases. In addition, the study will bring new important and detailed data on the use of SGLT2 inhibition at an early stage in a risk population where pharmaceutical intervention is rare. As such, the results from this trial will challenge current medical practice and form the basis for futureclinical trials focused on diabetes prevention.

Contributors KF, FP and MEJ conceived the idea and designed the study. KF sponsors the trial and owns the data. MEJ is principal investigator. HA and LBN are coinvestigators. MR- $L$ and KK have contributed to the design of the exercise intervention. KF has drafted the manuscript. All authors have read and approved the final version of the manuscript.

Funding This work was initiated by Kristine Færch, MSc PhD, Steno Diabetes Center A/S. The study is funded by an unrestricted grant from the Novo Nordisk Foundation as an investigator-initiated study. The research project is conducted with support from AstraZeneca AB, Ascensia Diabetes Care, the Danish Innovation Foundation, and the University of Copenhagen. Mathias Ried-Larsen is funded by a postdoc grant from the Danish Diabetes Academy (supported by the Novo Nordic Foundation).

Disclaimer The funders have no role in study design, data collection and analysis, decision to publish, or preparation of manuscripts.

Competing interests KF, HA, LBN, FP and MEJ are employed by Steno Diabetes Center, a research hospital working in the Danish National Health Service. Until 31 December 2016 Steno Diabetes Center has been owned by Novo Nordisk A/S and has received part of its core funding from unrestricted grants from the Novo Nordisk Foundation and Novo Nordisk A/S. KF and MEJ own shares in Novo Nordisk A/S. KF, FP and MEJ have received research support from AstraZeneca. FP reports having received research grant from Novartis and lecture fees from MSD, AstraZeneca, Novo Nordisk, Novartis, Eli Lilly and Boehringer Ingelheim, and have served as a consultant for AstraZeneca and MSD.

Ethics approval Ethics Committee of the Capital Region $(\mathrm{H}-15011398)$ and the Danish Medicines Agency (EudraCT number: 2015-001552-30).

Provenance and peer review Not commissioned; externally peer reviewed.

Open Access This is an Open Access article distributed in accordance with the Creative Commons Attribution Non Commercial (CC BY-NC 4.0) license, which permits others to distribute, remix, adapt, build upon this work non-commercially, and license their derivative works on different terms, provided the original work is properly cited and the use is non-commercial. See: http://creativecommons.org/ licenses/by-nc/4.0/

(C) Article author(s) (or their employer(s) unless otherwise stated in the text of the article) 2017. All rights reserved. No commercial use is permitted unless otherwise expressly granted.

\section{REFERENCES}

1. Færch K, Vistisen D, Johansen NB, et al. Cardiovascular risk stratification and management in pre-diabetes. Curr Diab Rep 2014;14:493

2. Perreault L, Færch K. Approaching pre-diabetes. J Diabetes Complications 2014:28:226-33.

3. Knowler WC, Barrett-Connor E, Fowler SE, et al. Reduction in the incidence of type 2 diabetes with lifestyle intervention or metformin. N Engl J Med 2002;346:393-403.

4. Diabetes Prevention Program Research Group. HbA1c as a predictor of diabetes and as an outcome in the diabetes prevention program: a randomized clinical trial. Diabetes Care 2015;38:51-8.

5. Færch K, Borch-Johnsen K, Holst JJ, et al. Pathophysiology and aetiology of impaired fasting glycaemia and impaired glucose tolerance: does it matter for prevention and treatment of type 2 diabetes? Diabetologia 2009;52:1714-23.

6. Færch K, Hulman A, Solomon T. Heterogeneity of Pre-diabetes and type 2 Diabetes: implications for prediction, Prevention and treatment responsiveness. Curr Diab Rev 2015;12:30-41.

7. Færch K, Johansen NB, Witte DR, et al. Relationship between insulin resistance and $\beta$-cell dysfunction in subphenotypes of prediabetes and type 2 diabetes. J Clin Endocrinol Metab 2015;100:707-16.

8. Fizelova M, Stančáková A, Lorenzo C, et al. Glycated hemoglobin levels are mostly dependent on nonglycemic parameters in 9398 finnish men without diabetes. J Clin Endocrinol Metab 2015;100:1989-96.

9. Kanat M, Winnier D, Norton L, et al. The relationship between (beta)-cell function and glycated hemoglobin: results from the veterans administration genetic epidemiology study. Diabetes Care 2011;34:1006-10.

10. Brownlee M, Hirsch IB. Glycemic variability: a hemoglobin A1c-independent risk factor for diabetic complications. JAMA 2006;295:1707.

11. Monnier L, Mas E, Ginet C, et al. Activation of oxidative stress by acute glucose fluctuations compared with sustained chronic hyperglycemia in patients with type 2 diabetes. JAMA 2006;295:1681

12. Ceriello A, Esposito K, Piconi L, et al. Oscillating glucose is more deleterious to endothelial function and oxidative stress than mean glucose in normal and type 2 diabetic patients. Diabetes 2008;57:1349-54.

13. Torimoto K, Okada $\mathrm{Y}$, Mori $\mathrm{H}$, et al. Relationship between fluctuations in glucose levels measured by continuous glucose monitoring and vascular endothelial dysfunction in type 2 diabetes mellitus. Cardiovasc Diabetol 2013;12:1-7.

14. Su G, Mi S, Tao H, et al. Association of glycemic variability and the presence and severity of coronary artery disease in patients with type 2 diabetes. Cardiovasc Diabetol 2011;10:19-9.

15. Service FJ, Molnar GD, Rosevear JW, et al. Mean amplitude of glycemic excursions, a measure of diabetic instability. Diabetes 1970;19:644-55.

16. Downie E, Craig ME, Hing S, et al. Continued reduction in the prevalence of retinopathy in adolescents with type 1 diabetes: role of insulin therapy and glycemic control. Diabetes Care 2011;34:2368-73.

17. Probstfield JL, Hirsch I, O'Brien K, Investigators TF-ST, et al. Design of FLAT-SUGAR: randomized Trial of Prandial insulin Versus Prandial GLP-1 receptor agonist together with basal insulin and metformin for High-Risk type 2 Diabetes. Diabetes Care 2015;38:1558-66.

18. Færch K, Vaag A, Witte DR, et al. Predictors of future fasting and 2-h post-OGTT plasma glucose levels in middle-aged men and womenthe Inter99 study. Diabet Med 2009;26:377-83.

19. Healy GN, Dunstan DW, Shaw JE, et al. Beneficial associations of physical activity with $2-\mathrm{h}$ but not fasting blood glucose in australian adults: the AusDiab study. Diabetes Care 2006;29:2598-604.

20. Assah FK, Ekelund U, Brage S, et al. Free-living physical activity energy expenditure is strongly related to glucose intolerance in cameroonian adults independently of obesity. Diabetes Care 2009;32:367-9. 
21. Færch K, Vaag A, Holst JJ, et al. Natural history of insulin sensitivity and insulin secretion in the progression from normal glucose tolerance to impaired fasting glycemia and impaired glucose tolerance: the Inter99 study. Diabetes Care 2009;32:439-44

22. Van Dijk JW, Manders RJ, Canfora EE, et al. Exercise and 24-h glycemic control: equal effects for all type 2 diabetes patients? Med Sci Sports Exerc 2013;45:628-35.

23. Colberg SR, Sigal RJ, Fernhall B, et al. Exercise and type 2 diabetes: the American College of Sports Medicine and the American Diabetes Association: joint position statement. Diabetes Care 2010;33:e147-e167.

24. Ross R, Hudson R, Stotz PJ, et al. Effects of exercise amount and intensity on abdominal obesity and glucose tolerance in obese adults: a randomized trial. Ann Intern Med 2015;162:325-34.

25. Karstoft K, Winding K, Knudsen SH, et al. The effects of free-living interval-walking training on glycemic control, body composition, and physical fitness in type 2 diabetic patients: a randomized, controlled trial. Diabetes Care 2013;36:228-36.

26. Jelleyman C, Yates T, O'Donovan G, et al. The effects of highintensity interval training on glucose regulation and insulin resistance: a meta-analysis. Obes Rev 2015;16:942-61.

27. Little JP, Francois ME. High-intensity interval training for improving postprandial hyperglycemia. Res Q Exerc Sport 2014;85:451-6.

28. Little JP, Jung ME, Wright AE, et al. Effects of high-intensity interval exercise versus continuous moderate-intensity exercise on postprandial glycemic control assessed by continuous glucose monitoring in obese adults. Appl Physiol Nutr Metab 2014;39:835-41.

29. Karstoft K, Clark MA, Jakobsen I, et al. The effects of 2 weeks of interval vs continuous walking training on glycaemic control and whole-body oxidative stress in individuals with type 2 diabetes: a controlled, randomised, crossover trial. Diabetologia 2017;60:1-10.

30. Ferrannini $E$. The target of metformin in type 2 diabetes. $N$ Engl $J$ Med 2014;371:1547-8.

31. Lehtovirta M, Forsén B, Gullström M, et al. Metabolic effects of metformin in patients with impaired glucose tolerance. Diabet Med 2001;18:578-83.

32. Li CL, Pan CY, Lu JM, et al. Effect of metformin on patients with impaired glucose tolerance. Diabet Med 1999;16:477-81.

33. Morel Y, Golay A, Perneger T, et al. Metformin treatment leads to an increase in basal, but not insulin-stimulated, glucose disposal in obese patients with impaired glucose tolerance. Diabet Med 1999;16:650-5.

34. Rasouli N, Kern PA, Reece EA, et al. Effects of pioglitazone and metformin on beta-cell function in nondiabetic subjects at high risk for type 2 diabetes. Am J Physiol Endocrinol Metab 2007;292:E35 9-E365.

35. Abdul-Ghani MA, DeFronzo RA. Lowering plasma glucose concentration by inhibiting renal sodium-glucose cotransport. $J$ Intern Med 2014;276:352-63.

36. Roden M, Weng J, Eilbracht J, et al. Empagliflozin monotherapy with sitagliptin as an active comparator in patients with type 2 diabetes: a randomised, double-blind, placebo-controlled, phase 3 trial. Lancet Diabetes Endocrinol 2013;1:208-19.

37. Ferrannini E, Berk A, Hantel S, et al. Long-term safety and efficacy of empagliflozin, sitagliptin, and metformin: an active-controlled, parallel-group, randomized, 78-week open-label extension study in patients with type 2 diabetes. Diabetes Care 2013;36:4015-21.

38. Ferrannini E, Muscelli E, Frascerra S, et al. Metabolic response to sodium-glucose cotransporter 2 inhibition in type 2 diabetic patients. J Clin Invest 2014;124:499-508.

39. Polidori D, Sha S, Ghosh A, et al. Validation of a novel method for determining the renal threshold for glucose excretion in untreated and canagliflozin-treated subjects with type 2 diabetes mellitus. $J$ Clin Endocrinol Metab 2013;98:E867-E871.

40. Sha S, Devineni D, Ghosh A, et al. Canagliflozin, a novel inhibitor of sodium glucose co-transporter 2 , dose dependently reduces calculated renal threshold for glucose excretion and increases urinary glucose excretion in healthy subjects. Diabetes Obes Metab 2011:13:669-72.

41. Polidori D, Sha S, Mudaliar S, et al. Canagliflozin lowers postprandial glucose and insulin by delaying intestinal glucose absorption in addition to increasing urinary glucose excretion: results of a randomized, placebo-controlled study. Diabetes Care 2013;36:2154-61.

42. Hussey EK, Clark RV, Amin DM,et al. Single-dose pharmacokinetics and pharmacodynamics of sergliflozin etabonate, a novel inhibitor of glucose reabsorption, in healthy volunteers and patients with type 2 diabetes mellitus. J Clin Pharmacol 2010;50:623-35.

43. Lidegaard L.P HA-LS, Johansen NB, Witte DR, et al. Role of physica activity energy expenditure versus estimated fitness level in impaired glucose regulation. Diabetologia 2015.

44. American Diabetes Association. 5. Prevention or Delay of Type 2 Diabetes. Diabetes Care 2017;40:S44-S47.

45. American Diabetes Association. Standards of medical care in diabetes--2014. Diabetes Care 2014;37:14-80.

46. Lipska KJ, Bailey CJ, Inzucchi SE. Use of metformin in the setting of mild-to-moderate renal insufficiency. Diabetes Care 2011;34:1431-7.

47. Hinnen D. Glucuretic effects and renal safety of dapagliflozin in patients with type 2 diabetes. Ther Adv Endocrinol Metab 2015;6:92-102.

48. Bandura A. Self-efficacy: toward a unifying theory of behavioral change. Psychol Rev 1977;84:191-215.

49. Ministry of Environment and Food in Denmark. The danish official dietary recommendations. secondary the danish official dietary recommendations. http://altomkost.dk/raad-og-anbefalinger/deofficielle-kostraad/

50. EasyTrial - an online web-based clinical trial management system. secondary EasyTrial - an online web-based clinical trial management system. $2016 \mathrm{http}: / / w w w . e a s y t r i a l . n e t /$

51. Zinman B, Wanner C, Lachin JM, et al. Empagliflozin, Cardiovascular Outcomes, and mortality in type 2 Diabetes. N Engl J Med 2015;373:2117-28.

52. Lamanna C, Monami M, Marchionni N, et al. Effect of metformin on cardiovascular events and mortality: a meta-analysis of randomized clinical trials. Diabetes Obes Metab 2011;13:221-8.

53. Hanefeld M, Sulk S, Helbig M, et al. Differences in glycemic variability between normoglycemic and prediabetic subjects. J Diabetes Sci Technol 2014;8:286-90.

54. Wang YM, Zhao LH, Su JB, Y-m W, L-h Z, J-b S, et al. Glycemic variability in normal glucose tolerance women with the previous gestational diabetes mellitus. Diabetol Metab Syndr 2015;7:1-8.

55. Ma CM, Yin FZ, Wang R, et al. Glycemic variability in abdominally obese men with normal glucose tolerance as assessed by continuous glucose monitoring system. Obesity 2011;19:1616-22.

56. Kim HS, Shin JA, Lee SH, et al. A comparative study of the effects of a dipeptidyl peptidase-IV inhibitor and sulfonylurea on glucose variability in patients with type 2 diabetes with inadequate glycemic control on metformin. Diabetes Technol Ther 2013;15:810-6.

57. Fang FS, Gong YP, Li CL, Cl L, et al. Comparison of repaglinide and metformin monotherapy as an initial therapy in Chinese patients with newly diagnosed type 2 diabetes mellitus. Eur $\mathrm{J}$ Endocrinol 2014;170:901-8.

58. Henry RR, Rosenstock J, Edelman S, et al. Exploring the potential of the SGLT2 inhibitor dapagliflozin in type 1 diabetes: a randomized, double-blind, placebo-controlled pilot study. Diabetes Care 2015;38:412-9. 